\title{
Lexicografía electrónica y traducción: propuesta de un modelo de adquisición de estrategias
}

\author{
$M^{a}$ DEL MAR SÁNCHEZ RAMOS \\ Universidad de Alcalá
}

El diccionario es a la vez amigo y enemigo del traductor; puede ayudar y puede obstaculizar la labor traductora. Es una obra de referencia a la que todo traductor, como profesional del lenguaje, recurre. No obstante, y a pesar del indudable valor tanto lingüístico como extralingüístico (Alvar Ezquerra 1981), desde los estudios de traducción se nos asegura que el interés prestado a esta obra de consulta no está en consonancia con su papel decisivo en el proceso de trasvase. Posiblemente, y como indica Roberts (1997), no se presta la merecida atención debido a la falta de conocimiento que se tiene de los productos lexicográficos y de su uso. A su vez, somos testigos de la ineludible presencia de las nuevas tecnologías. Es por ello, y conscientes de que un buen manejo de diccionarios y nuevas tecnologías puede reportar innumerables beneficios a nuestros discentes en relación a sus lenguas de trabajo (inglés y español en nuestro caso), que proponemos en este artículo un modelo de enseñanza y adquisición de estrategias de uso de diccionarios electrónicos para el traductor. Veremos, en primer lugar, los pasos iniciados desde la lexicografía pedagógica para pasar, posteriormente, a los estudios y recomendaciones que abogan por una instrucción en el uso de los diccionarios por parte del traductor y, por extensión, de los profesionales de la lengua. Seguiremos con una descripción breve de los diccionarios electrónicos y concluiremos con nuestra propuesta de estrategias de consulta de diccionarios electrónicos. Si bien se parte del traductor como principal usuario, el mencionado modelo, sin duda, puede adaptarse al usuario del diccionario en general con el fin de beneficiarse de una instrucción que consideramos imprescindible en el ámbito académico.

\section{LEXICOGRAFÍA PEDAGÓGICA Y TRADUCCIÓN: OPINIONES Y PERSPECTIVAS}

La falta de instrucción o el desconocimiento de obras lexicográficas adecuadas son algunos de los problemas que los traductores y usuarios de diccionarios en general aducen a la hora de hablar del diccionario como herramienta de consulta. Así, para solventar estas dificultades, surgió uno de los campos lingüísticos más prometedores y disciplina a caballo entre enseñanza y elaboración de diccionarios: la lexicografía pedagógica, donde autores de la talla de Hartmann (2001) se han encargado de otorgarle el reconocido prestigio que se merece. Sus orígenes se remontan al año 1935, cuando los lingüistas West y Endicott, movidos por su afán de recopilar lecturas simplificadas, asentaron las bases para la elaboración de un diccionario dirigido a estudiantes, si bien 
este no era su objetivo final. Su trabajo fue el germen de los tradicionales listados de palabras y supuso el punto de partida para los futuros recuentos léxicos que se utilizarían en las definiciones de las distintas entradas de un diccionario. A ellos también se deben las convenciones que hoy en día se tienen en cuenta a la hora de confeccionar un diccionario monolingüe (Rundell 1998). Apenas indaguemos en la literatura especializada, esta disciplina se encuentra ya asentada en campos como la enseñanza de segundas lenguas, pero su evolución ha sido más escasa en los estudios de traducción. Nuestro objetivo es, por tanto, contribuir a estrechar lazos entre traducción y lexicografía desde una de sus vertientes, como es la enseñanza de su uso y, en concreto, el uso y el manejo de diccionarios electrónicos, obras imprescindibles en el traductor del siglo XXI.

Gelpí Arroyo (1999) señala que «un diccionario es, ante todo y por su objetivo natural, un producto lingüístico, pero es también un producto histórico, cultural, social, temporal, económico e ideológico». Esta definición condensa en tres líneas la ingente información que un diccionario «entrega» a un traductor, entre otros usuarios. De un lado, el uso del diccionario facilita la comprensión del texto original. De otro, posibilita la reescritura de un texto en la lengua meta. No obstante, a menudo el traductor se muestra reticente a usar el diccionario. Hay diversas razones: falta de instrucción en su uso y conocimiento lexicográfico (Roberts 1997) o complejo de inferioridad (Silversteyn 2001). Creemos que con una lexicografía en constante avance, parece recomendable que el traductor, más que luchar contra los diccionarios, se alíe con ellos de manera pragmática y eficaz. Esta instrucción se hace aún más necesaria en el momento que nos ha tocado vivir, donde es imprescindible el domino de las nuevas tecnologías para mejorar la calidad del producto traducido y no menguar su calidad.

Distintos estudios se han aproximado de forma descriptiva a las relaciones entre diccionario y traducción. Mackintosh (1998) comenta que pueden encontrarse investigaciones en las que se incluyen tareas de traducción como una de las actividades examinadas (Tomaszyzcyk 1979; Atkins y Varantola 1998). Sin embargo, estos estudios no son un fiel reflejo del panorama general ya que en algunos de ellos se investiga el uso de los diccionarios en tareas de traducción pero no el comportamiento de los traductores o estudiantes de traducción. La necesidad de estudios específicamente centrados en traductores profesionales o noveles adquiere una mayor importancia si tenemos en cuenta que la capacidad de conocer y usar las obras de referencia se considera un elemento imprescindible dentro de las distintas definiciones de la competencia traductora. Además, el diccionario es una de las obras de consulta más inmediatas para el traductor y una de las que mayor tiempo y esfuerzo suponen.

Los estudios no son muy abundantes y no es hasta finales de los 80 cuando se atisba cierto interés por este tipo de investigaciones. Autores de la talla de Meyer $(1988,1990)$ y Roberts (1990) investigan el uso del diccionario, concretamente el diccionario bilingüe, por parte de los discentes en tareas de traducción inversa y directa. Tras estos primeros pasos, siguen estudios que de forma rigurosa y científica han centrado su atención el alumno de traducción como usuario de diccionarios. Tal es el caso de los trabajos de Starren y Thelen (1990), Mackintosh (1998), Varantola (1998) Atkins y Varantola (1998), Forget (1999), Corpas et al. (2001), Livbjerg y Mees (2003), Sanchez Ramos (2007), Hartmann (2007), Law (2009). Todos ellos tienen en común entre sus objetivos integrar el 
uso del diccionario en el proceso de traducción y enseñar estrategias de consulta. Otros incluso van más allá y proponen un diccionario híbrido que reúna las características de los diccionarios monolingües y bilingües (Mackintosh 1998). Para concluir este apartado, nos quedamos con las palabras de Corpas et al. (2001: 255), donde claramente aboga por una mayor instrucción lexicográfica en los estudios de traducción y afirma: «se [...] debería incluir una parte dedicada exclusivamente a los diccionarios, ubicándola dentro de las distintas fuentes de documentación a disposición del traductor e intérprete».

\section{DICCIONARIOS ELECTRÓNICOS}

Mucho se ha escrito sobre las diferencias entre los diccionarios en formato papel y diccionarios eléctronicos (Forget 1999; Heuberger 2000; Krajka 2004). Uno de los estudios seminales en cuanto a parámetros de diferenciación y caracterización de los diccionarios electrónicos frente a los diccionarios en formato papel, lo encontramos en Forget (1999), centrado en analizar en detalle diccionarios monolingües en francés, monolingües en inglés y bilingües inglés-francés/francés-inglés existentes en la época de realización de su trabajo. Forget (1999), sin embargo, no tiene en cuenta los diccionarios disponibles en Internet o en línea, si bien ambos comparten gran parte de las características. Como se desprende del estudio de Forget (1999), la principal diferencia entre los diccionarios electrónicos en general y los diccionarios en formato papel se encuentra en la organización de la información, el acceso a la misma y la información adicional que ofrecen al usuario; y es por estas características por las que han revolucionado el ámbito educativo y se constituyen como una de las herramientas lexicográficas imprescindibles del siglo XXI. De manera muy breve, la organización del contenido léxico, mucho mayor que la de un diccionario en formato papel, se fundamenta en el hipertexto y los modelos de organización hipertextual. (Fernández-Pampillón Cesteros y Matesanz del Barrio 2003), lo que permite una estructura asociativa del contenido, ya que prácticamente todos los elementos del diccionario electrónico están relacionados. En segundo lugar, en cuanto a la accesibilidad al contenido léxico, hay que destacar las variadas técnicas de accesibilidad y posibilidades de búsqueda (Pastor y Alcina 2010) Las características apuntadas en el apartado anterior (hipertextualidad, interactividad, etc.) sin duda facilitan la estructuración y acceso al contenido macroestructural y microestructural del diccionario electrónico. Por un lado, estas herramientas incluyen, al igual que los diccionarios en formato papel, información macroestructural (información sobre el diccionario, guía de uso, lista de abreviaturas, información variada, guía de pronunciación, etc.), pero la diferencia radica en la presentación de esta información macroestructural, apoyada en su gran mayoría por las características hipertextuales. La forma en la que se expone toda esta información es diversa, lo que sin duda aporta rapidez y eficacia en la consulta, siempre y cuando se muestre por parte del usuario cierta destreza en el manejo de estas nuevas herramientas. Estas características también pueden aplicarse a la microestructura. Las entradas de los diccionarios electrónicos muestra prácticamente la misma información léxica que un diccionario en formato papel (pronunciación, significado, uso, ejemplos, etc.), pero es la presentación lo que realmente diferencia el contenido de uno y otro diccionario, como hemos comentado anteriormente. Por último, junto con la información macroestructural y mi- 
croestructural, los diccionarios electrónicos pueden ofrecer herramientas adicionales destinadas a facilitar y consolidar las búsquedas. Esos recursos adicionales dependerán del tipo de diccionario electrónico (CD-ROM o en línea). Así, pueden encontrarse enlaces a documentos para practicar el manejo de los distintos diccionarios, secciones dedicadas al comentario de distintas unidades léxicas, creación de glosarios, etc.

Con todo, el traductor de siglo XXI cuenta con nuevas herramientas de consulta, como es el diccionario electrónico, entre otras (Austermühl 2001; Bowker 2003; Calzada Pérez 2005). Creemos que la enseñanza de consulta de los diccionarios electrónicos puede aportar:

- Familiarización del alumnado de traducción con unas herramientas imprescindibles en su formación.

- Fomento de la autonomía del aprendizaje y la ampliación de conocimientos.

- Ampliación de los niveles léxicos.

- Uso de las palabras con una mayor precisión y propiedad.

- Eficacia y efíciencia en los procesos traductológicos.

3. Diseño DE UN MODElO DE ADQUiSICIÓN DE ESTRATEGIAS DE CONSULTA DE LOS DICCIONARIOS ELECTRÓNICOS

Siguiendo muy de cerca los estudios realizados y las recomendaciones de uso que se sugieren, presentamos a continuación un modelo de adquisición de estrategias focalizado en los diccionarios electrónicos (figura 1), que consideramos indispensables también en la formación contemporánea del traductor, debido, en gran parte, al avance de las nuevas tecnologías en el ámbito traductológico y a las ventajas que un uso adecuado de los mismos puede reportar al estudiante. Este modelo no es definitivo y se concibe como un indicador de investigación futura.

En primer lugar, para diseñar y poner en práctica esta propuesta didáctica, resulta imprescindible conocer el punto de partida de nuestros alumnos en cuanto a conocimiento léxico, estrategias de búsqueda y familiarización con las nuevas tecnologías y así diseñar las actividades que se consideren oportunas e indicadas para cada situación específica.

Como puede observarse en la fig. 1, nuestra propuesta didáctica integradora se desglosa en tres fases:

1. Fase instructiva inicial de uso de los diccionarios electrónicos, en la que los discentes se familiarizan con las prestaciones que ofrecen las diversas herramientas electrónicas.

2. Fase de adquisición de estrategias de uso de diccionarios electrónicos (monolingües en inglés y en español, bilingües inglés-español), que incide sobre aspectos léxicos concretos y sobre el uso de los diccionarios en el proceso de trasvase de dichas facetas léxicas. Como es sabido, todo traductor ha de poseer un dominio (mínimo) de la lengua de partida y de llegada, de ahí que consideremos de máxima importancia también la instrucción en diccionarios monolingües en español. 
3. Fase de reflexión y evaluación, en la que se prepara al alumno para que desarrolle una postura crítica y para que reflexione sobre su proceso de búsqueda y el producto traductor final.

DISEÑO DE UN MODELO INSTRUCTIVO DE ADQUISICIÓN DE ESTRATEGIAS DE CONSULTA DE LOS DICCIONARIOS ELECTRÓNICOS

\begin{tabular}{|c|c|c|c|c}
\hline $\begin{array}{c}\text { Familiarización del alumna- } \\
\text { TRUCTIVA } \\
\text { INICIAL }\end{array}$ & $\begin{array}{c}\text { con el material de trabajo } \\
\text { (diccionarios electrónicos) } \\
\text { Instrucción en su uso }\end{array}$
\end{tabular}
FASE DE AD- QUISICIÓN DE ESTRATEGIAS DE CONSUL-

Desarrollo de estrategias de consulta de los diccionarios electrónicos en los procesos traductológicos

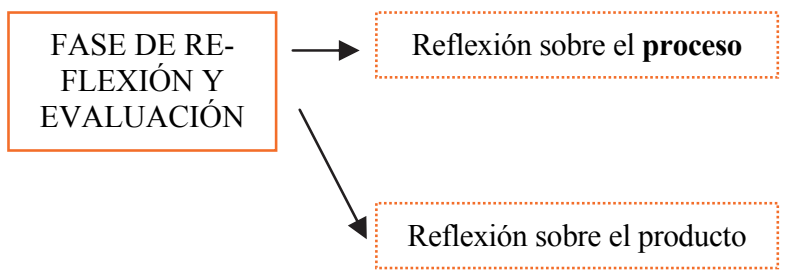

Fig. 1. Propuesta didáctica de adquisición de estrategias de consulta de diccionarios electrónicos A continuación, pasamos a describir cada una de estas fases con mayor de detalle.

\subsection{Fase instructiva inicial}

Los objetivos de esta fase inicial se concretan en:

1. Concienciar al alumnado de la importancia de conocer los distintos diccionarios electrónicos.

2. Ofrecer una instrucción precisa en cuanto a la estructura del contenido de los distintos diccionarios electrónicos.

3. Ofrecer una instrucción precisa para una correcta interpretación de la entrada de los distintos diccionarios electrónicos. 
4. Mostrar la utilidad de las herramientas adicionales que ofrecen los diccionarios electrónicos.

5. Ofrecer una pautas de consulta de los diccionarios.

Como vemos, de forma generalizada, esta primera fase se articula en torno al conocimiento de la tipología de diccionarios electrónicos y organización de su contenido. No hay duda de que la búsqueda será más eficaz si, en primer lugar, los estudiantes están familiarizados con los tipos de diccionarios e información de los mismos. Esto supone a priori un conocimiento o entrenamiento previo de estrategias relacionadas con las nuevas tecnologías. Tras esto, el conocimiento de la macro y microestructura de las herramientas electrónicas es el siguiente paso. A diferencia de los diccionarios en formato papel, en los que los estudiantes tienen que conocer la organización alfabética de las entradas, los diccionarios electrónicos, se caracterizan por ofrecer distintas posibilidades de búsqueda (Pastor y Alcina 2010). Gracias a la instrucción proporcionada en esta primera etapa, el alumno ya posee el andamiaje suficiente para poder interpretar toda la información que indica la entrada, la microestructura así como símbolos, la organización de las distintas acepciones o la la discriminación de la información. Detallamos un listado de estrategias propias de esta fase de instrucción:

- Conocer los distintos diccionarios electrónicos y clasificarlos (para luego decidir su uso) teniendo en cuanta las necesidades traductológicas.

- Analizar las características de los diccionarios electrónicos (organización de la información).

- Conocer las distintas posibilidades/opciones de búsqueda (búsqueda avanzada, búsqueda asistida, uso de comodines, etc.) de los diccionarios electrónicos.

- Conocer cómo funciona el sistema hipertextual de los diccionarios electrónicos.

- Conocer cómo usar la opción de «ayuda» de los diccionarios electrónicos.

- Distinguir donde se encuentra la información sobre uso del diccionario (guías de uso).

- Comprender las convenciones tipográficas, criterios de división de acepciones y uso símbolos y de abreviaturas de los diccionarios electrónicos.

- Conocer las herramientas adicionales de los diccionarios electrónicos y su funcionamiento.

- Dirigir las búsquedas atendiendo a las características de cada diccionario y a los problemas léxicos que se han de resolver en cada momento.

- Decidir cuándo utilizar el diccionario.

- Reflexionar sobre el proceso de búsqueda.

Una vez que se ha recibido esta instrucción inicial y se han desarrollado e interiorizado estas estrategias, el alumno de traducción se encuentra preparado para comenzar la siguiente fase, que denominamos fase de adquisición de estrategias, en la que se trabaja con aspectos léxicos que pueden ser fuente de dificultades a la vez que se desarrollan estrategias de consulta para facilitar la resolución de las dudas. 


\subsection{Fase de adquisición de estrategias de búsqueda}

Esta es una fase importante ya que en ella se pretende que el alumno sea capaz de sacar el máximo partido a toda la información que contiene un diccionario y, de este modo, mejorar el proceso de trasvase. Los objetivos de esta segunda fase son:

1. Desarrollar conocimientos léxicos en las dos lenguas implicadas en el proceso de traducción (inglés y español).

2. Desarrollar estrategias de búsqueda en los diccionarios electrónicos para solucionar problemas léxicos concretos.

3. Poner en práctica los conocimientos adquiridos en la primera fase.

4. Utilizar la información contextual para deducir el significado de las palabras.

5. Concienciar al alumnado de la importancia del diccionario monolingüe en la lengua de partida.

Todo diccionario ofrece al traductor información valiosa sobre la forma, que el traductor ha de saber detectar de manera rápida y consciente para solventar problemas lingüísticos. Podemos encontrar en los diccionarios (electrónicos) información sobre la categoría gramatical de las palabras, el comportamiento sintáctico y otras peculiaridades morfosintácticas. De este modo, pretendemos que el alumno de traducción adquiera las siguientes estrategias relacionadas con la forma de las unidades léxicas:

- Interpretar la transcripción fonética de las palabras.

- Identificar variaciones en cuanto a la pronunciación.

- Distinguir los acentos primarios y secundarios.

- Identificar la separación de sílabas.

- Identificar variaciones en la ortografía en la lengua de partida y de llegada.

- Reconocer los afijos más comunes y detectar cómo se muestran en un diccionario.

- Interpretar de manera eficiente y eficaz la información sintáctica y gramatical relevante para la elección de las acepciones apropiadas.

Los traductores y, en general, todos los usuarios de las obras de referencia, acuden al diccionario principalmente en busca de significado. Por ello, en esta fase de adquisición, conviene que el alumno también aprenda, entre otras cosas, a discriminar entre distintos tipos de significado. Entre las estrategias que pueden consolidarse ahora se encuentran: 
- Examinar en detalle la entrada del diccionario.

- Identificar el equivalente adecuado mediante las herramientas electrónicas de consulta (especialmente bilingües).

- Percibir, junto con el significado referencial y diferencial, el significado que las distintas palabras adquieren en función del contexto (connotativo, cotextual, contextual y metafórico).

- Reconocer las relaciones semánticas de las palabras (polisemia, sinonimia, antonimia, etc.).

- Utilizar los diccionarios monolingües en la lengua de partida para detectar matices de significado.

- Clasificar las distintas unidades léxicas que pertenecen al mismo campo semántico.

Por último, en la fase de desarrollo, hemos de contemplar un apartado para el uso del léxico en cotexto y en contexto. Estas nociones son de máxima importancia en el campo traductológico ya que influyen de manera decisiva en el trasvase hasta tal punto que, a menudo, sin ellos, la traducción resulta completamente imposible. En primer lugar, atendiendo al cotexto, cabe recordar que el léxico de una lengua no está formado sólo por palabras aisladas sino también por compuestos léxicos (locuciones, modismos, frases hechas) que adquieren un sentido unitario. Esto supone el fomento de unas estrategias específicas asociadas al uso de léxico en cotexto:

- Leer las definiciones y examinar en detalle los ejemplos para reconocer las posibles combinaciones sintácticas de las palabras.

- Examinar en detalle definiciones y ejemplos para identificar la información sobre las colocaciones.

- Identificar expresiones idiomáticas.

- Utilizar las funciones de búsqueda de los diccionarios electrónicos para buscar modismos y demás expresiones institucionalizadas.

- Poner en práctica las distintas posibilidades de búsqueda para agilizar y perfilar las consultas de léxico en cotexto.

- Trasladar expresiones idiomáticas a la lengua meta con la ayuda del diccionario monolingüe en inglés.

El diccionario no sólo puede reportar beneficios en cuanto al uso del léxico en cotexto sino que, además, proporciona información de relieve sobre la utilización del léxico en contexto. Para acceder a dicha información con éxito puede impulsarse el aprendizaje de, entre otras, las siguientes estrategias: 
- Interpretar las etiquetas que los diccionarios ofrecen sobre las variaciones lingüísticas de uso (registro) y usuario (dialecto).

- Demostrar flexibilidad productiva al proporcionar distintos tipos de traducción.

- Interpretar las etiquetas que los diccionarios ofrecen sobre el uso de las palabras en determinados géneros, discursos y textos.

- Seleccionar los sinónimos adecuados para conseguir la cohesión en un texto.

- Compaginar la información contextual con el uso del diccionario para deducir el significado de las palabras.

Y junto con estrategias orientadas a la forma, al significado y al uso, podemos añadir otras cuantas estrategias, más generales, que completan los procesos de búsqueda del alumnado:

- Priorizar la búsqueda de léxico desconocido o dudoso.

- Practicar el trasvase a través de distintos tipos de textos: expositivos, instructivos y argumentativos.

- Desarrollar la creatividad léxica ante problemas concretos de traducción.

- Reformular el texto meta teniendo en cuanta las características de un texto específico (expositivos, instructivos y argumentativos).

- Desarrollar la intuición y el espíritu crítico ante posibles problemas de traducción.

\subsection{Fase de reflexión y evaluación}

Esta última fase es también de vital importancia en nuestro modelo ya que anima al alumno a reflexionar sobre el producto y el proceso desarrollados en este y así promover la autonomía del alumnado. La reflexión, por tanto, se aplica sobre las estrategias fomentadas durante las tres fases del aprendizaje (proceso) y sobre el conocimiento de aspectos concretos de léxico aprendidos en dichas fases (producto). Asimismo, se anima al estudiante a valorar — de forma crítica - la efectividad de la propuesta (y del programa didáctico que lo ejecuta). En este sentido, en la fase de reflexión se fomentan las siguientes estrategias:

- Reflexionar sobre el proceso de búsqueda.

- Ordenar las ideas aprendidas.

- Adoptar una postura crítica ante el uso de los diccionarios y valorar su eficacia.

- Valorar los conocimientos adquiridos.

- Valorar la metodología traductora propia.

- Aprender a responsabilizarse del aprendizaje propio.

\section{CONCLUSIONES}

Hemos visto a lo largo de este artículo la estrecha conexión que se establece entre la lexicografía y los estudios de traducción, aunque sigue siendo una parcela donde se reclama una mayor investigación. Con la llegada de las nuevas tecnologías y la falta de instrucción en cuanto al uso de diccionarios, se hace necesario una especificación de las estrategias de consulta necesarias para un uso correcto de las obras lexicográficas y, 
en concreto, de los diccionarios electrónicos. Así se examinaba, por un lado, los distintos estudios empíricos que trataban de dibujar el perfil de traductor como usuario de diccionarios y, por otro, las principales características de los diccionarios electrónicos. A la vista de todo lo especificado, exponíamos un modelo de adquisición de estrategias estructurado en tres fases (instructiva inicial, desarrollo y adquisición de estrategias y reflexión y evaluación). Esta propuesta, que ofrecemos como punto de partida y como un indicador para futuros estudios, persigue unos objetivos determinados, fomenta estrategias concretas y, en nuestra opinión, ofrece a la comunidad académica un punto de partida para estrechar lazos entre dos disciplinas tan relacionadas como la lexicografía y los estudios de traducción.

\section{REFERENCIAS BIBLIOGRÁFICAS}

Alvar EZQUERRA, Manuel (1981). «Los diccionarios bilingües: su contenido». Lingüística Española Actual, 3, 1, pp. 175-196

AtKInS, Sue y Krista VARANTOLA (1998): «Monitoring dictionary use», en S. Atkins, ed., Using Dictionaries, Tübingen, Niemeyer, pp. 83-122

AUSTERMÜHL, Frank (2001): Electronic Tools for Translators, Manchester, St. Jerome.

BOWKER, Lynne (2002): Computer-Aided Translation Technology, Ottawa, University of Ottawa Press.

CAlZADA PÉREZ, María (2005): «Corpus electrónicos como herramientas de documentación y formación de traductores», en D. Sales, eds., La Biblioteca de Babel: Documentarse para traducir, Granada, Comares, pp. 163-200.

CORPAS PASTOR, Gloria, Jorge LeIVA RoJo y Ma José VARela SALINAS (2001): «El papel del diccionario en la formación de traductores e intérpretes: análisis de necesidades y encuestas de uso», en M. Ayala Castro, ed., Diccionarios y enseñanza, Alcalá de Henares, Universidad de Alcalá, pp. 239-273.

FÉrnANDEZ-PAMPILlón Cesteros, Ana y María MATESANZ DEl BARrio (2003), «Los diccionarios electrónicos: hacia un nuevo concepto de diccionario», en C. López Alonso y A. Séré, eds., Nuevos géneros discursivos: los textos electrónicos, Madrid, Biblioteca Nueva, pp. 137-158.

Forget, Nadine (1999): Les Dictionnaires Électroniques dans l'optique de la Traduction, Thèse de maitise, École de traduction et d'interprétation, Ottawa, Université d'Ottawa, http://aix1.uottawa.ca/ etithese/nforg/frmain.htm [Consulta: 24/04/2012].

GelPí ARroyo, Cristina (1999): «Diccionarios del español en Internet»,Ar@cne. Revista de recursos en Internet sobre Ciencias Sociales, 50, http://www.ub.es/geocrit/arac50.htm [Consulta: 30/04/2012].

HARTMANN, Reirnhard (2001): Teaching and Researching Lexicography, Essex, Pearson Education.

(2007): Interlingual Lexicography. Selected Essays on Translation Equi-valence, Contrastive Linguistics and the Bilingual Dictionary, Lexicographica Series Maior 133, Tübingen: M. Niemeyer.

HEUBERGER, Reinhard (2000): Monolingual Dictionaries for Foreign Learners of English: A Constructivist Evaluation of the State-of-the-Art Reference Works in Book Form and 
on CD-ROM, Austrian Studies in English 87, Whilhem Braumüller, UniversitätsVerlagsbuchhandlng.

KRAJKA, Jaroslaw (2004): «Electronic dictionaries as teaching and learning toolsPosibilities and limitations», en M. C. Campoy Cubillo y P. Safont Jordá, eds., Computer-Mediated Lexicography in the Foreign Language Learning Context, Castellón, Universitat Jaume I, pp. 29-46.

LAW, Wai-on (2009): Translation students' use of dictionaries: a Hong Kong case study for Chinese to English translation, Tesis doctoral, Durham, Universidad de Durham, http://etheses.dur.ac.uk/729/ [Consulta: 24/04/2012].

LIVBJERG, Inge e Inger MEES (2003): «Patterns of dictionary use in non-domain-specific translation», en F. Alves, ed., Triangulating Translation, Amsterdam, John Benjamins, pp. 123-136.

MACKINTOSH, Kristen (1998): «An empirical study of dictionary use in L2-L1 translation», en S. Atkins, ed., Using Dictionaries, Tübingen, Niemeyer, pp. 123-149.

MEYER, Ingrid (1988): «The general bilingual dictionary as a working tool in theme», $\mathrm{Me}$ ta, 43, 3: 368-376.

(1990): «Interlingual meaning-text lexicography: towards a new type of dictionary for translation», en J. Steele, ed., The Meaning-Text Theory of Language: Linguistics, Lexicography, and Practical Implications, Ottawa, University of Ottawa Press, pp. 175-270.

PASTOR, Verónica y Amparo AlcinA (2010): «Search techniques in electronic dictionaries: a classification for translators», International Journal of Lexicography, 23, 3, pp. 307354.

RoBERTS, Roda (1990): «Translation and the bilingual dictionary», Meta, 35, 1, pp. 74- 81. (1992): «Translation pedagogy: strategies for improving dictionary use», TTR, 5, 1, pp. 49-76.

(1997): «Using dictionaries efficiently», Conferencia presentada en 38th Annual Conference of the American Translators Association, San Francisco, California. http://www.dico.uottawa.ca/articles-en.htm [Consulta: 30/04/2012].

RuNDELL, Michael (1998): «Recent trends in English pedagogical lexicography», International Journal of Lexicography, 11, 4, pp. 315-342.

SÁNCHEZ RAMOS, Ma del Mar (2007): «Aproximación empírica al uso de los diccionarios electrónicos y la adquisición de la competencia léxica traductora», Horizontes en Lingüistica Aplicada, 6, 2, pp. 273-294.

SiLVERSTEYN, Boris (2001): «The dictionary: my friend, my enemy», ATA Chronicle, 30, 1, pp. 52-57.

STARREN, Peter y Marcel THELEN (1990): «General dictionaries and students of translation: A report on the use of dictionaries in the translation process»), en T. Magay y J. Zigàny, eds., BudaLEX'88 Proceedings, Budapest, Akadémiai Kiadó, pp. 447-458.

TOMASZCZYK, Jerzy (1979): «Dictionaries: users and uses», Glottodidactica, 12, pp. 103119.

VARANTOLA, Krista (1998): «Translators and their use of dictionaries», en S. Atkins, ed., Using Dictionaries, Tübingen, Niemeyer, pp. 179-192. 